\title{
Super-Hydrides of Lanthanum and Yttrium: On Optimal Conditions for Achieving near Room Temperature Superconductivity
}

\author{
Hans Hermann Otto \\ Materialwissenschaftliche Kristallographie, Clausthal University of Technology, Clausthal-Zellerfeld, Germany \\ Email: hhermann.otto@web.de
}

How to cite this paper: Otto, H.H. (2019) Super-Hydrides of Lanthanum and Yttrium: On Optimal Conditions for Achieving near Room Temperature Superconductivity. World Journal of Condensed Matter Physics, 9, 22-36.

https://doi.org/10.4236/wjcmp.2019.91002

Received: January 15, 2019

Accepted: February 18, 2019

Published: February 21, 2019

Copyright $\odot 2019$ by author(s) and Scientific Research Publishing Inc. This work is licensed under the Creative Commons Attribution International License (CC BY 4.0).

http://creativecommons.org/licenses/by/4.0/

\begin{abstract}
Recently, many seminal papers deal with the syntheses, stability and superconducting properties of super-hydrides like $\mathrm{LaH}_{10}$ or $\mathrm{YH}_{10}$ under high pressure, reporting critical temperatures near room temperature. In the first run one will assume that the involved metal atoms contribute a number of 3 electrons to the pairing pool corresponding to their valence. However, another possibility may be that the cationic valence is somewhat smaller, for instance only 2.29 , resulting in a nominal electron number per cation of $\sigma_{0}=0.229 \approx$ $3 / 13$ instead of 0.3 . Then, we will have a numerical equality to the optimum hole number in the cuprate high- $T_{\mathrm{c}}$ superconductors, a number that reflects the fractal nature of electronic response in superconductors. However, if one still keeps up the oxidation state of +3 of lanthanum, one will need 13 hydrogen atoms to match the optimum $\sigma_{0}$. Such composition may be found at the phase boundary between the observed $\mathrm{LaH}_{10}$ and $\mathrm{LaH}_{16}$ phases. Partial ionic replacement is suggested to shift the super-hydride composition into the $\sigma_{0}$ optimum. Micro-structural phenomena such as multiple twinning and ferroelastic behavior as observed with cuprates may also influence the superconductivity of super-hydrides. Finally, epitaxial growth of super-hydrides onto a specially cut diamond substrate is proposed.
\end{abstract}

\section{Keywords}

Superconductivity, Super-Hydride, Clathrate, $\mathrm{LaH}_{10}, \mathrm{YH}_{10}$, Faujasite,

High Pressure, Optimum of Pairing Charge Carriers, Slab Width,

Ionic Substitution, Epitaxial Growth, Diamond Substrate, Fractality

\section{Introduction}

Excitingly, different groups of outstanding researchers independently synthe- 
sized and investigated super-hydrides of lanthanum respectively yttrium, and found superconducting transition temperatures only less below room temperature with $T_{\mathrm{c}}$ around $250 \mathrm{~K}$, when applying high pressure [1] [2] [3] [4]. The mankind's dream of room temperature superconductivity could thus be realized in near future. Since the suggestion of Ashcroft [5] that metallic hydrogen might be a room temperature superconductor, research on hydrogen and hydrogen-rich compounds in this direction had already led to many promising results. A milestone of such research was the observation of conventional superconductivity at $203 \mathrm{~K}$ at high pressure in the $\operatorname{Im} \overline{3} \mathrm{~m}$ structure of $\left(\mathrm{H}_{2} \mathrm{~S}\right)_{2} \mathrm{H}_{2}$ [6] [7] [8]. Whereas the proposal of the present author to upload $\mathrm{H}_{3} \mathrm{~S}$ in the cavities of a zeolite to reduce the applied high pressure could not be realized as yet [9], we now see the new superb results obtained by dense ordering of hydrogen in a clathrate structure centered by large metal ions like $\mathrm{La}^{3+}$ or $\mathrm{Y}^{3+}$, if we still use the usual valence. In this short contribution we pose the question, how many electrons per hydrogen within the super-hydride actually contribute to the conventional superconducting pairing scenario and whether the electronic response in such superconductors is of fractal nature.

Looking at the recent results, then a guide for experimenters was given by the published relationship between mean cationic charge and critical temperature, when extrapolating the depicted branch for conventional superconductors of Figure 1 in Reference [10] to small values.

Many other researchers have contributed to the growing field of super-hydrides, and the reader may follow their valuable work summarized in the references above.

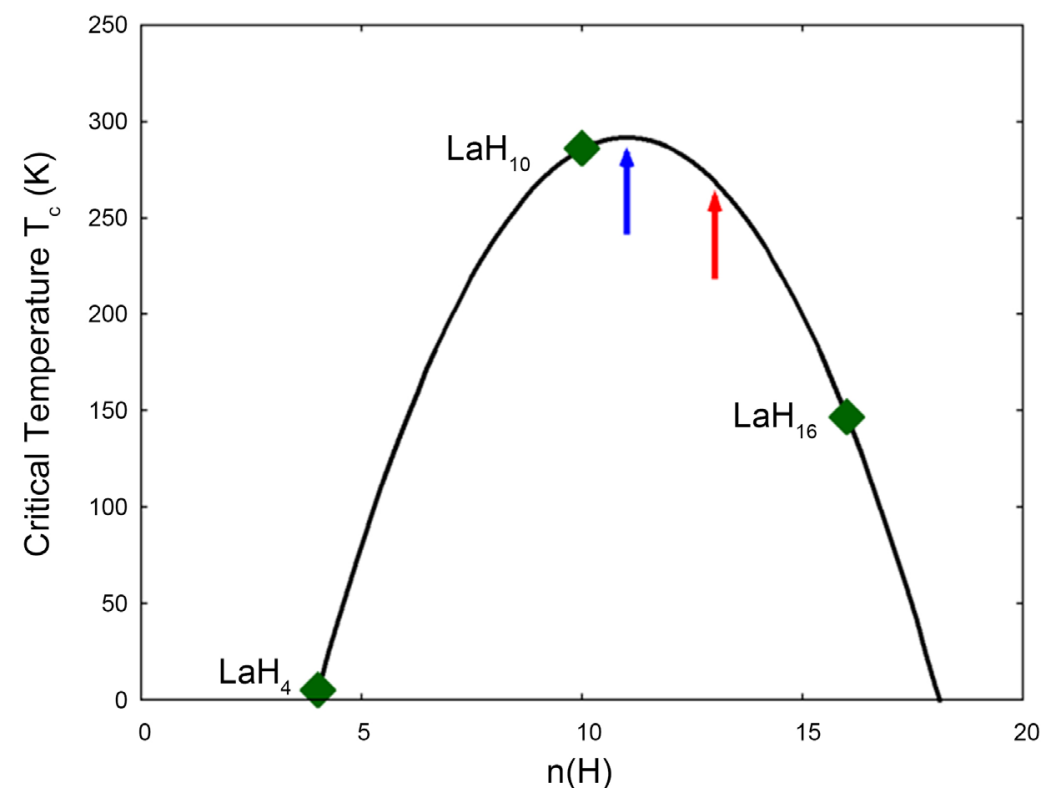

Figure 1. Critical temperature $T_{\mathrm{c}}$ versus the hydrogen atom number $n(\mathrm{H})$ of lanthanum hydrides approximated by a parabola according to Semenok et al. [1]. The blue arrow depicts the maximum of the parabola with $T_{\mathrm{c}}=292 \mathrm{~K}$ at a composition of about $\mathrm{LaH}_{11}$. The red arrow marks the assumed point at $\mathrm{LaH}_{13}$. 
This article deals with the question of the optimal charge of pairing electrons in super-hydrides of lanthanum and yttrium and discusses the influence of the width of structural layers. It furthermore proposes chemical substitutions to enhance superconductivity and suggests suitable substrates for epitaxial growth of super-hydrides to reduce the applied pressure. Attention is drawn to micro-structural phenomena, possible multiple twinning of super-hydride phases or the role of interfaces. Finally, the beauty of the 32-membered hydrogen cage around lanthanum in $\mathrm{LaH}_{10}$ is looked at separately, where the structure projection down the [111] direction gives a near-perfect Kagome pattern.

\section{The Optimum of Pairing Charge Carriers}

Turning first to unconventional superconductivity of the high- $T_{\mathrm{c}}$ cuprates, an optimum number of holes resulted in the unique number $\sigma_{0}=0.229$. This number can also be confirmed for the family of FeAs-based superconductors [11]. Some time ago, the present author connected this optimum with Hardy's quantum entanglement probability $\varphi^{5}$ [12] [13]

$$
\sigma_{0}=\frac{8}{\pi} \varphi^{5}=0.2296,
$$

where $\varphi=(\sqrt{5-1}) / 2=0.6180339887 \ldots$ is the golden mean.

The interpretation of a fractal nature of electronic response in superconductors is consistent with generated $d$-wave fractal patterns in unconventional superconductors as a consequence of antiferromagnetism, documented by scanning tunneling microscopy [14] [15] [16].

The fractal-hierarchical structure of electrons entangled in pairs obviously determines this optimum near a quantum critical point that can be linked also with the universal fractal constant $\delta_{1}=8.7210972 \ldots$ of the renormalized quadratic Hénon map (remember the quadrilateral layer structure of the cuprates or the tetragonal respectively cubic crystal structures of the super-hydrides) [10] [13]

$$
\sigma_{0} \approx \frac{2}{\delta_{1}}=0.2293 \text {. }
$$

Despite the differences in theory and experiment between conventional and unconventional superconductors we should search for similarities between them regarding the perfect tuning through the optimum numbers of charge carriers involved in their pairing. Turning to the super-hydrides formed by cages of hydrogen around large metal atoms like La. The usual oxidation state of lanthanum should be +3 . However, also the oxidation states of +1 respectively +2 have been observed for La bound to hydrogen [17] or hydrocarbons [18]. The nominal oxidation state could actually be less than +3 . If one tentatively selects the oxidation state of +2.29 instead of +3 , one reaches numerical equality of the spend electrons to the optimum number of holes in the cuprate high- $T_{\mathrm{c}}$ superconductors, a number that reflects the fractal nature of electronic response in superconductors. However, if one still keeps up the +3 oxidation state, $\sigma_{0}$ electrons per 
hydrogen atoms can be adapted, when their number would be 13 , because $\sigma_{0}$ can be represented as a quotient of the two Fibonacci numbers 3 and 13

$$
\sigma_{0} \approx 3 / 13
$$

Two phases of composition $\mathrm{LaH}_{10}$ and $\mathrm{LaH}_{16}$ have been experimentally confirmed [2]. One the boundary between both phases a nominal composition of about $\mathrm{LaH}_{13}$ may actually exist. A topotactic intergrowth of the (111) plane of cubic $\mathrm{LaH}_{10}$ or the (001) plane of rhombohedral $\mathrm{LaH}_{10}$ with the (001) plane of hexagonal $\mathrm{LaH}_{16}$ is likely, possibly connecting the two phases by a common 6-ring of hydrogen atoms. Also multiple twinning as frequently observed in zeolites can lead to the required composition. The a-axis lattice parameters for rhombohedral $\mathrm{LaH}_{10}$ and hexagonal $\mathrm{LaH}_{16}$ are nearly identical (3.66 ̊̊ compared to $3.68 \AA$ [19]).

Semonov et al. [1] illustrated the critical temperature $T_{c}$ versus the hydrogen atom number $n(\mathrm{H})$ of lanthanum hydrides by a parabola. The maximum of the parabola yielded a composition of about $\mathrm{LaH}_{11}$ (Figure 1). This is a step towards the composition of $\mathrm{LaH}_{13}$ as the intended optimum.

The few experimental points can also be represented by an asymmetric Gaussian or Cauchy distribution function [20] with a smooth slope on the side of less hydrogen compared to the steeper slope on the hydrogen-rich side to shift the curve maximum towards the $\mathrm{LaH}_{12}$ or even $\mathrm{LaH}_{13}$ composition. From density arguments this is the more likely solution.

In Figure 2 earlier results [10] that illustrate the dependence between the critical temperature and the mean cationic charge $\left\langle q_{c}\right\rangle$ for unconventional superconductors were completed with data points for the lanthanum super-hydrides respectively $\mathrm{H}_{3} \mathrm{~S}$, showing the branch of $n$-type superconductors besides the $p$-type branch. The blue curve fits the $\sigma>\sigma_{0}$ branch of $n$-type superconductors according to the simple relation

$$
\begin{gathered}
T_{c}(K) \propto(447.8 \pm 16.7) \cdot e^{-\sigma} \\
\text { or finer adjusted } T_{c}(K) \propto(553.8 \pm 18.3) /\left(e^{\sigma}+\frac{8}{7} \cdot \sigma\right) .
\end{gathered}
$$

If one calculates the average charge over all atoms, avoiding in the case of super-hydrides the terms cation or counterion, then for $\mathrm{LaH}_{12}$ the mean charge would yield the optimum of $\sigma=\sigma_{0}=0.23$.

However, it remains open how pure hydrogen under very high pressure can be treated within this scheme. Molecular dissociation of $\mathrm{H}_{2}$ near $500 \mathrm{GPa}$ could lead to superconductivity around $356 \mathrm{~K}$ [21]. From Equation $4 \mathrm{a}$ an optimum transition temperature of $T_{\mathrm{c}} \approx 356 \mathrm{~K}$ for super-hydrides can be estimated matching well the predicted one for pure hydrogen.

The mean cationic charge $\left\langle q_{c}\right\rangle$ (in units of holes) of unconventional superconductors is limited to the asymptotic value of (see Figure 2)

$$
<q_{c}>_{\text {asymp }} \approx \frac{13}{\delta_{1}}
$$




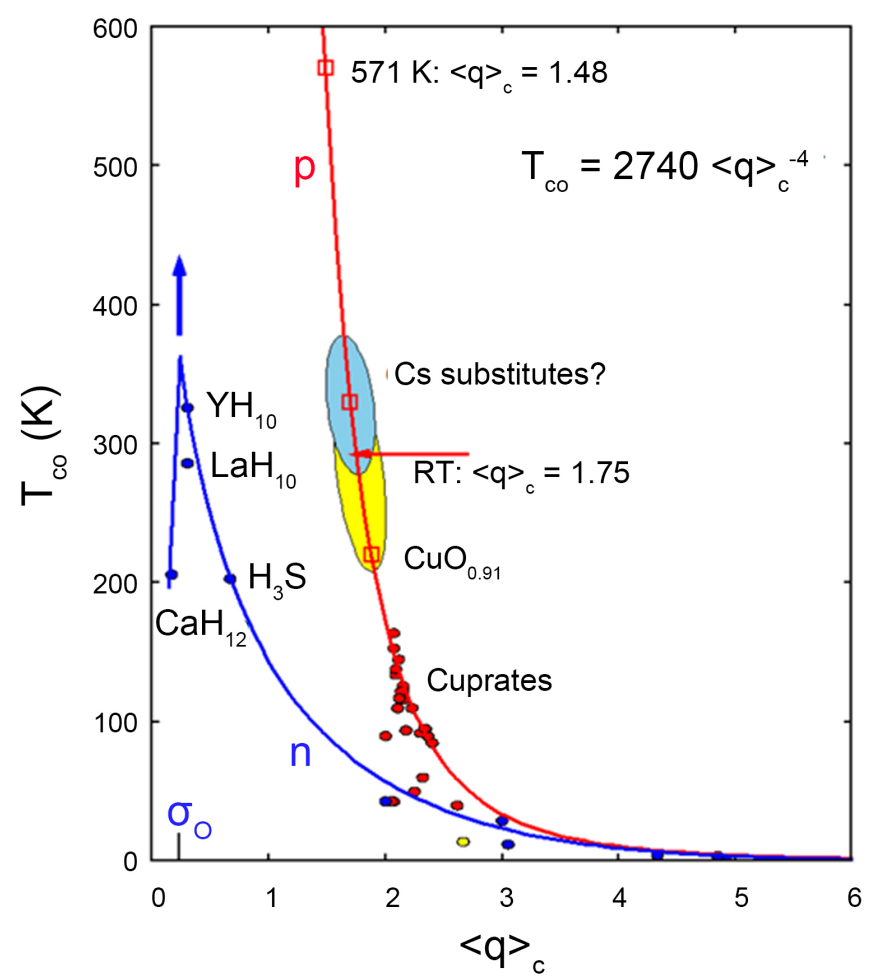

Figure 2. Mean cationic charge versus critical temperature of superconducting compounds. $p$ : $p$-type branch, $n$ : $n$-type branches having $\sigma<\sigma_{0}$ and $\sigma>\sigma_{0}$ ( $\sigma$ equivalent to $\left\langle q_{\mathrm{c}}\right\rangle$ ). For more details see [10].

This may underline once again the fractal nature of unconventional superconductivity.

\section{Dependence of the Critical Temperature on the Widths of Structural Slabs}

The critical temperature of unconventional superconductors is obviously inversely proportional to the distance of copper atoms in the $\mathrm{CuO}_{2}$ plaquette [11]. On the basis of this specification the present author argued in a previous publication [10] that room temperature superconductivity could be reached with a smart structure where the relevant atomic distances have been reduced by a factor of $\sqrt{2}$ compared to cuprates giving about $3.85 \AA / \sqrt{2} \approx 2.72 \AA$.

Indeed, the super-hydrides are such smart compounds. This can be verified with crystallographic data given by Kruglov et al. [19] respectively Semenok et al. [1].

In Table 1 relevant atomic distances are presented for important structural slabs in super-hydrides of lanthanum, subjected to a pressure of $150 \mathrm{GPa}$. However, these distances are strongly pressure dependent.

If one focuses on such small slab distances, the smallest one is found for $\mathrm{LaH}_{10}$ with a very high expected critical temperature around $286 \mathrm{~K}$. Only $\mathrm{YH}_{10}$ is reported to have higher $T_{\mathrm{c}}$ than $\mathrm{LaH}_{10}$. This may be explained with the pronounced smaller ionic radius of $\mathrm{Y}^{3+}$ compared to $\mathrm{La}^{3+}$ and therefore an even 
Table 1. Lattice parameters respectively slab widths for lanthanum super-hydrides.

\begin{tabular}{|c|c|c|c|c|}
\hline Phase & Space Group & Lattice Parameters $[\AA]$ & Slab Width $[\AA]$ & Reference \\
\hline \multirow{2}{*}{$\mathrm{LaH}_{2}$} & \multirow{2}{*}{$\mathrm{P} 6 / \mathrm{mmm}$} & $a=2.80$ & 2.80 & \multirow{2}{*}{ [19] } \\
\hline & & $c=2.72$ & 2.72 & \\
\hline \multirow{2}{*}{$\mathrm{LaH}_{3}$} & \multirow{2}{*}{$\mathrm{Cmcm}$} & $a=2.74$ & \multirow{2}{*}{2.74} & \multirow{2}{*}{ [19] } \\
\hline & & $c=6.03$ & & \\
\hline \multirow{3}{*}{$\mathrm{LaH}_{4}$} & \multirow{3}{*}{$\mathrm{I} 4 / \mathrm{mmm}$} & $a=2.76$ & \multirow{3}{*}{$\begin{array}{l}2.74 \\
2.80\end{array}$} & \multirow{3}{*}{ [19] } \\
\hline & & $b=10.69$ & & \\
\hline & & $c=2.80$ & & \\
\hline \multirow{2}{*}{$\mathrm{LaH}_{10}$} & \multirow{2}{*}{$\mathrm{R} \overline{3} \mathrm{~m}$} & $a=3.66$ & \multirow{2}{*}{$c / 3=2.84$} & \multirow{2}{*}{ [19] } \\
\hline & & $c=8.53$ & & \\
\hline $\mathrm{LaH}_{10}$ & $\mathrm{Fm} \overline{3} \mathrm{~m}$ & $a=5.1019$ & 2.551 & [1] \\
\hline $\mathrm{LaH}_{12}$ & $\mathrm{Fd} 3 \mathrm{~m}$ & $a=6.64$ & $a / 2=3.32 \quad a \cdot \sqrt{2} / 4=2.35$ & This work \\
\hline \multirow{2}{*}{$\mathrm{LaH}_{16}$} & \multirow{2}{*}{$\mathrm{P} 6 / \mathrm{mmm}$} & $a=3.68$ & \multirow{2}{*}{3.68} & \multirow{2}{*}{ [19] } \\
\hline & & $c=3.70$ & & \\
\hline
\end{tabular}

smaller slab width for $\mathrm{YH}_{10}$. The effective ion radius of $\mathrm{Y}^{3+}$ under ambient conditions is $r\left(\mathrm{Y}^{3+}\right)=1.011 \AA$ [22] compared to $r\left(\mathrm{La}^{3+}\right)=1.15 \AA$. The quotients of $T_{\mathrm{c}}$ values compared to the reciprocal of the corresponding radii yield similar values

$$
T_{\mathrm{c}}\left(\mathrm{YH}_{10}\right) / T_{\mathrm{c}}\left(\mathrm{LaH}_{10}\right)=1.132 ; r\left(\mathrm{La}^{3+}\right) / r\left(\mathrm{Y}^{3+}\right)=1.137
$$

The change of ionic radii due to high hydrogen coordination is compensated by the use of their ratio. For comparison, the slab width for superconducting H3S in the $\operatorname{Im} \overline{3} \mathrm{~m}$ space group (No 229), lattice parameter $a=2.9840 \AA$ [6], is estimated to be $\sqrt{3} \cdot a / 2=2.5842 \AA$.

The proportionality of $T_{\mathrm{c}}$ can be recast in a very simple relation when considering structural slabs $\mathrm{d}$ besides the mean charge $\sigma$ (supporting data see Addendum).

$$
T_{c}(K) \propto 1030 \cdot e^{-\sigma} \cdot d^{-1} .
$$

An analysis of the pre-factor with a dimension discussion will be given in a forthcoming paper, including the Fermi speed of the charge carriers, the permittivity of the compounds and the Compton wavelength. The relation (7) is displayed in Figure 3.

\section{Expose Chemical Pressure to Reduce Hydrostatic One}

Developments in future could be approaches to reduce the too high pressure by growing the clathrate super-hydride clysters on a suitable, but inert surface structure with lattice parameters slightly less than that of the clathrate itself to generate chemical pressure. The crystal structure of $\mathrm{LaH}_{10}$ was reported to be cubic in the $\mathrm{Fm} \overline{3} \mathrm{~m}$ space group with a lattice parameter of $a=5.1019$ (5) $\AA$ [2]. Alloys of Ta with Os as single crystals would deliver a substrate having the required lattice distances [23] but do also form hydrides under pressure. So it would be only by chance to find a suitable substrate material. However, why should not use diamond as an inert substrate? The ambient pressure lattice parameter of diamond 


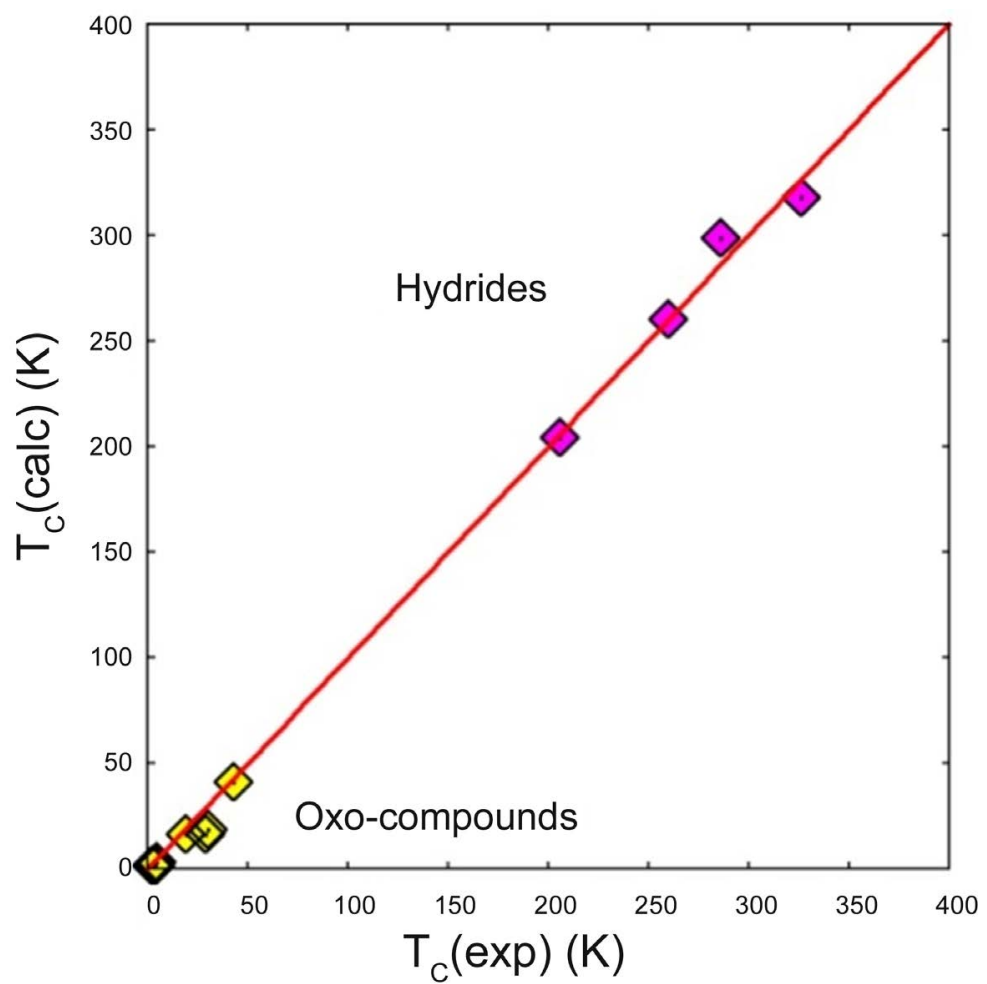

Figure 3. Plot of experimental critical temperatures of conventional superconductors versus calculated ones according to Equation (7).

is $a=3.56679 \AA$, giving for the [110] direction an atomic distance of $\sqrt{2} \cdot a=$ $5.0442 \AA$. Then the task is to find a promising distance in a perpendicular direction. Travelling 2 identity periods in the $a_{2}$ direction and 5 ones in the $a_{3}$ direction, you find identity at $19.2078 \AA=4 \cdot 4.8019 \AA$ distance. When cutting a (110) diamond plate at an angle of $21.8^{\circ}$, you can make a promising substrate. The cut is parallel to the $(2 \overline{2} 5)$ diamond lattice plan. Besides, assuming that a lower synthesis pressure is needed by epitaxial growth, one could work with a less elaborated pressure apparatus. The pressure dependence of the lattice parameter of diamond up to $40 \mathrm{GPa}$ can be found in reference [24].

\section{Partial Atomic Replacement}

Besides this possibility the partial substitution of $\mathrm{La}^{3+}$ or $\mathrm{Y}^{3+}$ by the comparably large $\mathrm{Ca}^{2+}$ ion may be considered to study the effected trend. The ionic radius of eight-coordinated $\mathrm{Y}^{3+}$ seems to be the most promising with $r_{[8]}=1.011 \AA$ [22]. A fortunate substitution can produce internally chemical pressure to reduce the applied external pressure. If one combines $\mathrm{YH}_{10}$ and $\mathrm{CaH}_{12}$ in equal atomic parts (no phase separation expected), then all possible combinations of the mean charge of $q=2.5$ with the number $n(\mathrm{H})$ of $\mathrm{H}$ atoms would shift the $\sigma=q / n(\mathrm{H})$ values towards the proposed optimum of $\sigma_{0}$. The result could be a superconductor with a critical temperature fairly above room temperature synthesized by well reduced pressure.

Finally, one finds thallium with a question mark in reference [1]. However, 
$\mathrm{Tl}^{3+}$ (less toxic than $\mathrm{Tl}^{1+}$ ) should be interesting too because of its similar ion radius and ionic charge compared to $\mathrm{Y}^{3+}$, when the $\mathrm{Tl}^{3+}$ oxidation state is stable to hydrogen under high pressure. A gain in the superconducting response of the order of the ionic radii ratio of $r\left(\mathrm{Y}^{3+}\right) / r\left(\mathrm{Tl}^{3+}\right)=1.011 / 0.89=1.136$ may be suggested compared to $\mathrm{YH}_{10}$.

The optimum charge per hydrogen may also be accomplished through the partial fluoride replacement or interstitial addition according to $\mathrm{LaH}_{9.1} \mathrm{~F}_{0.9}$ or $\mathrm{LaH}_{10} \mathrm{~F}_{0.7}$ that resulted for both cases in $\sigma=0.23$. However, the high formation energy of $\mathrm{LaF}_{3}$ will make such substitution unlikely.

\section{Comparing Unconventional Superconductors with Conventional Ones}

In view of the same cations that are liable to generate very high critical temperatures, a mutual stimulation of the research between unconventional superconductors and conventional ones could be thought of. Common of both compound classes are the same optimal number of electrons or holes and the inverse proportionality to structural slab widths respectively $\mathrm{CuO}_{2}$ plaquette distances. The same optimal cationic charge and inverse proportionality to structural units such as $\mathrm{CuO}_{2}$ plaquettes respectively slab widths are common for both substance classes. The occurrence of multiple twinning together with ferroelastic behavior, observed incuprates, may be suspected even with super-hydrides, despite their high symmetry [25]. Ferroelastic forces causing micro-domain or nano-domain formation can strongly influence electric conductivity and pathways for charge transport [25]. Electrons can be sucked towards the domain boundaries.

After all, there are promising cage structures in both classes, for instance the superconducting bucky ball compounds [26] besides the suggested antiferromagnetic cuprate super-cage with possibly induced carrier delocalization [27]. Let us continue with examples of cage structures, as a supplement to previously predicted or synthesized hydrogen clathrates. A faujasite-like $\mathrm{LaH}_{12}$ or $\mathrm{YH}_{12}$ clathrate structure, grown onto a suitable substrate, is considered to be near the possible optimum of electronic charge. The faujasite prototype consists of sodalite cages (truncated octahedra, $\left[4^{6} 6^{8}\right]$ ) distanced by sixfold double rings (polyeder notation $\left[4^{6} 6^{2}\right]$ ), whereas the cages in $\mathrm{LaH}_{10}$ are not "sodalite-like", but consist of $\left[4^{6} 6^{12}\right]$ polyhedra, where such truncated dodecahedra are further connected by four-membered double rings [19]. When relating the density to the number of hydrogen atoms of the cage structures for lanthanum super-hydrides as displayed in Figure 4, one can estimate $D_{\mathrm{x}}=6.84 \mathrm{~g} \cdot \mathrm{cm}^{-3}$ for an assumed composition of $\mathrm{LaH}_{12}$. Then the cubic lattice parameter for an assumed face-centered faujasite-type $\mathrm{LaH}_{12}$ super-hydride with space group $\mathrm{Fd} \overline{3} \mathrm{~m}$ and $\mathrm{Z}=4$ formula units would yield

$$
a=\sqrt[3]{\frac{Z \cdot M}{D_{x} \cdot N_{L}}}=6.64 \AA,
$$

where $M$ is the molar weight and $N_{\mathrm{L}}$ is Loschmidt's constant. A general site with 


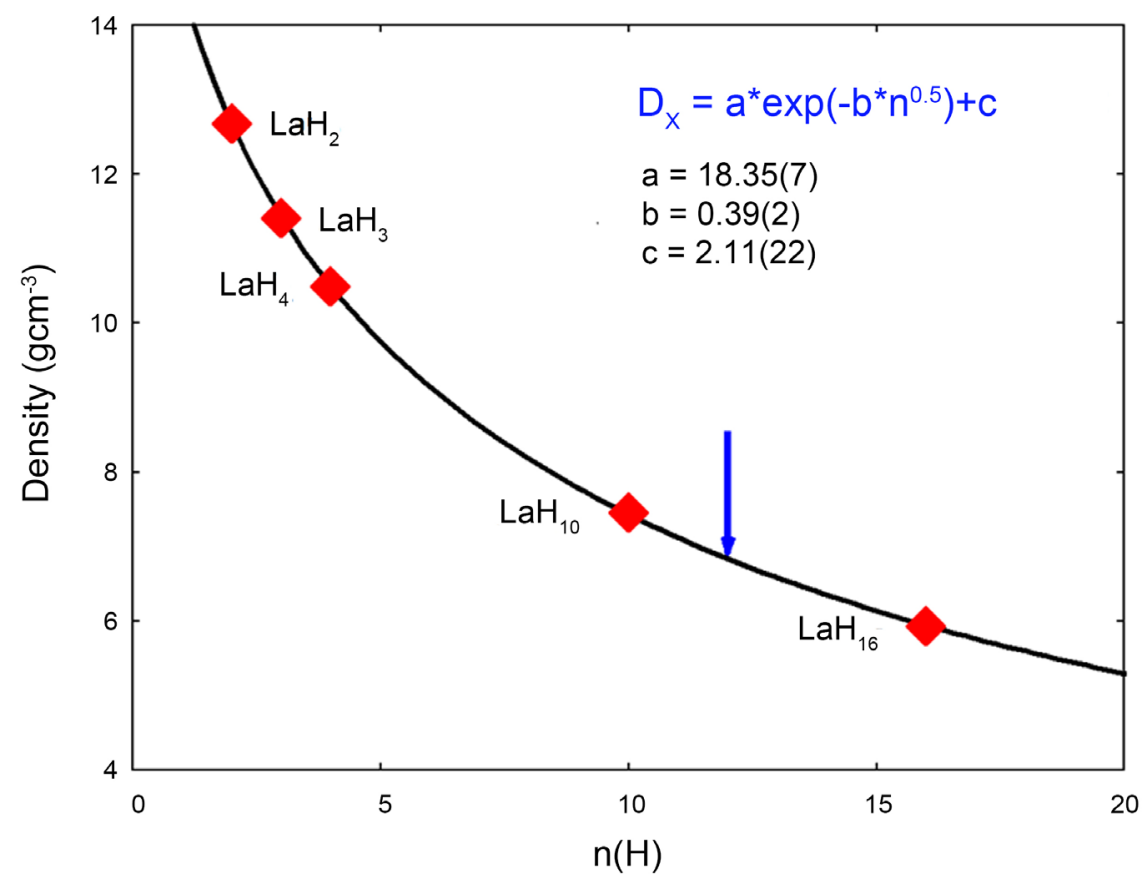

Figure 4. Calculated density $D_{\mathrm{x}}\left(\mathrm{g} \cdot \mathrm{cm}^{-3}\right)$ versus number of hydrogen atoms $n(\mathrm{H})$ for lanthanum super-hydrides. The relation was used to estimate the density of a possible $\mathrm{LaH}_{12}$ compound (blue arrow).

multiplicity 92 would be occupied by hydrogen. Mean distances between hydrogen atoms were found to be $0.84 \AA$. However, if evaluating superconducting properties, this structure type may less fortunate than the $\mathrm{LaH}_{10}$ one. When considering the number of available electrons per hydrogen together with the width of the structural slab of $d=3.32 \AA$, a possible critical temperature of about $260 \mathrm{~K}$ can be estimated. However, if choosing the smaller value of $d=2.35 \AA$, the critical temperature would yield about $340 \mathrm{~K}$.

Another cage structure, the silicon clathrate $\mathrm{Ba}_{7.2} \mathrm{Si}_{46}$ [28], may be a candidate to try a fortunate substitution to check whether superconductivity could be reached, if one substitutes half of the $\mathrm{Ba}^{2+}$ by $\mathrm{K}^{+}$or $\mathrm{Rb}^{+}$to adapt the optimum of $\sigma_{0}$. In addition, the present author recommends the synthesis of $\mathrm{La}_{3.5} \mathrm{Si}_{46}$ or $\mathrm{La}_{4.6} \mathrm{C}_{60}$.

Besides the great success with super-hydrides, experimental physicists should not lose sight of the further development of unconventional superconductors via routes proposed earlier [10], reinvestigating the effect of filamentary superconductivity up to $220 \mathrm{~K}$ observed in oriented multiphase $\mathrm{Y}-\mathrm{Ba}-\mathrm{Cu}-\mathrm{O}$ thin films [29] [30] respectively onto $\mathrm{CuO}-\mathrm{Cu}$ interfaces [31] [32]. Could gaseous impurities such as hydrogen be responsible for the effect?

\section{On the Beauty of the $\left[4^{6} 6^{12}\right]$ Cage of $\mathrm{LaH}_{10}$}

If one deals with polyhedral structures, the symbol $\left[\Sigma p_{i}^{f_{i}}\right]$ is used, denoting a convex polyhedron with polygon multiplicities $p_{i}$ of faces $f_{i}$. The number of edges $e$ of the polyhedron counts as 


$$
\Sigma e=\frac{1}{2} \Sigma p_{i} f_{i}
$$

Applying Euler's topologic invariant for convex polyhedra [33],

$$
\Sigma c+\Sigma f-\Sigma e=2
$$

the number of corners $c$ yields [27]

$$
\Sigma c=\frac{1}{2} \Sigma p_{i} \cdot f_{i}-\Sigma f_{i}+2 .
$$

Using the polyhedron symbol $\left[4^{6} 6^{12}\right]$ for $\mathrm{LaH}_{10}$ one counts $\Sigma c=32$ hydrogen atoms around the lanthanum ion. From number theory this beautiful cage with 32 corners is highly interesting due to the fact that the number 32 is near the Fibonacci number 33 . With this assessment, this prototypic cubic cage could be considered as being near chaos. In case of the observed $\mathrm{LaH}_{10+\mathrm{x}}$ variant [1], the extra hydrogen may distort the cage towards a more fractal network. Strikingly, if the crystal structure of $\mathrm{LaH}_{10}$ is projected down the [111] direction, an almost perfect Kagome lattice is formed with a tiling distance of $a^{\prime}=\sqrt{3} \cdot a / 8=1.105 \AA$ (dominant $\mathrm{H}-\mathrm{H}$ distance), where $a=5.1019 \AA$ is the cubic lattice parameter.

The $\mathrm{LaH}_{10}$ cage structure, optimized by few ionic replacements, is the recommended candidate to reach best superconducting properties, but $\mathrm{YH}_{6}$ is interesting too because of lower synthesis pressure [34]. Whether the proposed $\mathrm{LaH}_{12}$ structure could deliver higher $T_{c}$ values depends on synthesis progress.

\section{Conclusions}

Large cations such as $\mathrm{Ba}^{2+}$ or $\mathrm{La}^{3+}$ play an important but different role in superconducting materials. Whereas the large $\mathrm{Ba}^{2+}$ in cuprates, for instance, is able to accumulate hole carriers in its vicinity [35], lanthanum ions spend optimum electrons for the pairing, and deliver suitable $\mathrm{H}-\mathrm{H}$ distances when densely surrounded with hydrogen in a cagy structure. Thereby, the oxidation state of La may possibly be less than it is suggested by the usual valence of $3+$. However, if one maintains the usual oxidation state, one needs 13 hydrogen atoms surrounding the cation to fulfill the assumed condition for optimal superconductivity, represented by the optimum number of electrons spend per hydrogen, $\sigma_{0} \approx$ 3/13. A nominal composition of $\mathrm{LaH}_{12}$ or even $\mathrm{LaH}_{13}$ may be existent onto the phase boundary between the observed phases $\mathrm{LaH}_{10}$ and $\mathrm{LaH}_{16}$, respectively. The number $\sigma_{0}$ reflects excellently the fractal nature of the electronic response leading to superconductivity. Therefore, some ionic substitutions were recommended to reach optimum superconducting results by trying to synthesize not pure but more complex super-hydrides. Synthesis options were recommended such as $\mathrm{La}_{0.5} \mathrm{Ca}_{0.5} \mathrm{H}_{10}, \mathrm{Y}_{0.5} \mathrm{Ca}_{0.5} \mathrm{H}_{10}$ or $\mathrm{Y}_{0.3} \mathrm{Na}_{0.7} \mathrm{H}_{6}$. In addition, a potential faujasite-type super-hydride structure of composition $\mathrm{LaH}_{12}\left(\mathrm{YH}_{12}\right)$ was proposed.

The difference in the critical temperature between $\mathrm{LaH}_{10}\left(T_{\mathrm{c}}=286 \mathrm{~K}\right)$ and $\mathrm{YH}_{10}\left(T_{\mathrm{c}}=326 \mathrm{~K}\right)$ was traced back to the inverse dependence of the superconductive response from widths of dominant structural slabs. Furthermore, epitaxial growth of the hydrogen clathrates of $\mathrm{La}(\mathrm{Y})$ onto a $(2 \overline{2} 5)$ diamond sub- 
strate is proposed to reduce the applied high pressure.

Last but not least, the potentially fractal nature of the phenomenon superconductivity, coined by the author [10] [13], has been documented by various hints.

\section{Conflicts of Interest}

The author declares no conflicts of interest regarding the publication of this paper.

\section{References}

[1] Semenok, D.V., Kruglov, I.A., Kvashnin, A.G. and Oganov, A.R. (2018) On Distribution of Superconductivity in Metal Hydrides. arXiv: 1806.00865 [cond-mat.supr.con], 1-19, S1-S26.

[2] Drozdov, A.P., Kong, P.P., Minkov, V.S., Besedin, S.P., Kuzovnikov, M.A., Mozaffari, S., Balicas, L., Balakirev, F., Graf, D., Pragapenka, V.B., Greenberg, E., Knyazev, D.A., Tkacz, M. and Eremets, M.I. (2018) Superconductivity at $250 \mathrm{~K}$ in Lanthanum Hydride under High Pressures. arXiv: 1812.01561v1 [cond-mat.supwe-con], 1-16.

[3] Geballe, Z.M., Liu, H., Mishra, A.K., Ahart, M., Somayazulu, M., Meng, Y., Baldini, M. and Hemley, R.J. (2018) Synthesis and Stability of Lanthanum Superhydrides. Angewandte Chemie International Edition, 57, 688-692. https://doi.org/10.1002/anie.201709970

[4] Liu, H., Naumov, I.I., Geballe, Z.M., Somayazulu, M., Tse, J.S. and Hemley, R.J. (2018) Dynamics and Superconductivity in Compressed Lanthanum Superhydride. Physical Review, B98, Article ID: 100102. https://doi.org/10.1103/PhysRevB.98.100102

[5] Ashcroft, N.W. (1968) Metallic Hydrogen: A High-Temperature Superconductor. Physical Review Letters, 21, 1748-1749. https://doi.org/10.1103/PhysRevLett.21.1748

[6] Duan, D.F., Liu, Y.X., Tian, F.B., Li, D., Huang, X.L., Zhao, Z.L., Yu, H.Y., Liu, B.B., Tian, W.J. and Cui, T. (2014) Pressure-Induced Metallization of Dense $\left(\mathrm{H}_{2} \mathrm{~S}\right)_{2} \mathrm{H}_{2}$ with High- $\mathrm{T}_{\mathrm{c}}$ Superconductivity. Scientific Reports, 4, Article No. 6968.

[7] Drozdov, A.P., Eremets, M.L., Troyan, I.A., Ksenofontov, V. and Shylin, S.I. (2015) Conventional Superconductivity at $203 \mathrm{~K}$ at High Pressure. Nature, 525, 73-76. https://doi.org/10.1038/nature14964

[8] Drozdov, A.P., Eremets, M.I. and Troyan, I.A. (2014) Conventional Superconductivity at $190 \mathrm{~K}$ at High Pressure.

[9] Otto, H.H. (2015) Email Communication with Mikhail Eremets.

[10] Otto, H.H. (2016) A Different Approach to High- $\mathrm{T}_{\mathrm{c}}$ Superconductivity: Indication of Filamentary-Chaotic Conductance and Possible Routes to Superconductivity above Room Temperature. World Journal of Condensed Matter Physics, 6, 244-260. https://doi.org/10.4236/wjcmp.2016.63023

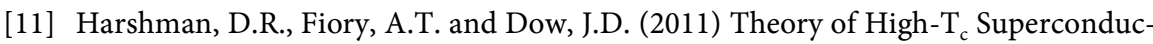
tivity: Transition Temperatures. Journal of Physics. Condensed Matter, 23, Article ID: 295701.

[12] Hardy, L. (1993) Nonlocality for Two Particles without Inequalities for Almost All Entangled States. Physical Review Letters, 71, 1665-1668.

https://doi.org/10.1103/PhysRevLett.71.1665 
[13] Otto, H.H. (2018) Reciprocity Relation between the Mass Constituents of the Universe and Hardy's Quantum Entanglement Probability. World Journal of Condensed Matter Physics, 8, 30-35. https://doi.org/10.4236/wjcmp.2018.82003

[14] Kohsaka, Y., Taylor, C., Fujita, K., Schmidt, A., Lupien, C., Hanaguri, T., Azuma, M., Takano, M., Eisaki, H., Takagi, H., Uchida, S. and Davis, J.C. (2007) An Intrinsic Bond-Centered Electronic Glass with Unidirectional Domains in Underdopedcuprates. Science, 315, 1380-1385. https://doi.org/10.1126/science.1138584

[15] Phillabaum, B., Carlson, E.W. and Dahmen, K.A. (2012) Spatial Complexity Due to Bulk Electronic Nematicity in a Superconducting Underdoped Cuprate. Nature Communications, Article No. 915. https://doi.org/10.1038/ncomms1920

[16] Sachdev, S. (2014) Quantum Entanglement \& Superconductivity. Public Lecture at the Perimeter Institute, Waterloo, ON.

[17] Ram, R.S. and Bernath, P.F. (1996) Fourier Transform Emission Spectroscopy of New Infrared System of LaH and LaD. Journal of Chemical Physics, 104, 6444-6451. https://doi.org/10.1063/1.471365

[18] Rosi, M. and Bauschlicher Jr., C.W. (1990) On the bonding of $\mathrm{La}^{+}$and $\mathrm{La}^{2+}$ to $\mathrm{C}_{2} \mathrm{H}_{2}$, $\mathrm{C}_{2} \mathrm{H}_{4}$, and $\mathrm{C}_{3} \mathrm{H}_{6}$. Chemical Physics Letters, 166, 189-194. https://doi.org/10.1016/0009-2614(90)87274-U

[19] Kruglov, I.A., Semenok, D.V., Szcęśniak, R., Esfahani, M.M.D., Kvashnin, A.G. and Oganov, A.R. (2018) Superconductivity in $\mathrm{LaH}_{10}$ : A New Twist of the Story. arXiv: 1810.01113, 1-28.

[20] Otto, H.H. (2018) Cauchy Functions Compared to the Gaussian for X-Ray Powder Diffraction Line Profile Fitting: An Exercise. Researchgate.net.

[21] McMahon, J.M. and Ceperley, D. (2011) High-Temperature Superconductivity in Atomic Metallic Hydrogen. Physical Review B, 84, 144515-144523. https://doi.org/10.1103/PhysRevB.84.144515

[22] Kim, D.J., Hyun, S.H. and Kim, S.G. (1994) Effective Ionic Radius of $\mathrm{Y}^{3+}$ Determined from Lattice Parameters of Fluorite-Type $\mathrm{HfO}_{2}$ and $\mathrm{ZrO}_{2}$ Solid Solutions. Journal of the American Ceramic Society, 77, 597-599. https://doi.org/10.1111/j.1151-2916.1994.tb07035.x

[23] Rudman, P.S. (1965) Lattice Parameters of Tantalum-Osmium Alloys. Journal of the Less Common Metals, 9, 77-79. https://doi.org/10.1016/0022-5088(65)90040-8

[24] Fujihisa, H., Sidorov, V.A., Takemura, K., Kanda, H. and Stishov, S.M. (1996) Pressure Dependence of the Lattice Constants of Diamond: Isotopic Effects. Journal of Experimental and Theoretical Physics Letters, 63, 83-88.

https://doi.org/10.1134/1.566982

[25] Otto, H.H. (2016) Perovskite Twin Solar Device with Estimated 50\% Bifacial PCE Potential and New Material Options. Researchgate.net, 1-8.

[26] Mitrano, M., Cantaluppi, A., Nicoletti, D., Kaiser, S., Peruchi, A., Lupi, S., Pietro, P., Pontiroli, D., Riccò, M., Clark, S.R., Jaksch, D. and Cavalleri, A. (2015) An Optically Stimulated Superconducting-Like Phase in $\mathrm{K}_{3} \mathrm{C}_{60}$ far above Equilibrium $\mathrm{T}_{c}$. arXiv: $1505.04529 \mathrm{v} 1,1-40$.

[27] Otto, H.H. (2015) Modeling of a Cubic Antiferromagnetic Cuprate Super-Cage. World Journal of Condensed Matter Physics, 5, 160-178. https://doi.org/10.4236/wjcmp.2015.53018

[28] Castillo, R., Schnelle, W., Bobnar, M., Burkhardt, U., Böhme, B., Baitinger, M., Schwarz, U. and Grin, Y. (2015) The Clathrate $\mathrm{Ba}_{8-x} \mathrm{Si}_{46}$ Revisited: Preparation Routes, Electrical and Thermal Transport Properties. Journal of Inorganic and 
General Chemistry, 641, 206-213.

[29] Djurek, D., Pretser, M., Knezovic, S., Drobac, D., Milat, O., Babic, E., Brnicevic, N., Furic, K., Medunic, Z. and Vukelja, T. (1987) Low Resistance State up to $210 \mathrm{~K}$ in a Mixed Compound Y-Ba-Cu-O. Physics Letters A, 123, 481-484. https://doi.org/10.1016/0375-9601(87)90349-5

[30] Schönberger, R., Otto, H.H., Brunner, B. and Renk, K.F. (1991) Evidence for Filamentary Superconductivity up to $220 \mathrm{~K}$ in Oriented Multiphase Y-Ba-Cu-O Thin Films. Physica C, 173, 159-162. https://doi.org/10.1016/0921-4534(91)90363-4

[31] Azzoni, C.B., Paravicini, G.B.A., Samoggia, G., Ferloni, P. and Parmigiani, F. (1990) Electric Instability in $\mathrm{CuO}_{1-x}$ : Possible Correlations with the $\mathrm{CuO}$-Based High Temperature Superconductors. Zeitschrift für Naturforschung A, 45, 790-794. https://doi.org/10.1515/zna-1990-0605

[32] Osipov, V.V., Kochev, I.V. and Naumov, S.V. (2010) Giant Electric Conductivity at the CuO-Cu Interface: HTSL-Like Temperature Variations. Journal of Experimental and Theoretical Physics, 93, 1082-1090. https://doi.org/10.1134/1.1427119

[33] Euler, L. (1752) Elementa Doctrine Solidorum. Novi Commentarii Academiae Scientiarum Imperialis Petropolitanae, 4, 109-160.

[34] Heil, C., di Cataldo, S., Bachelet, G.B. and Boeri, L. (2019) Superconductivity in Sodalite-Like Yttrium Hydride Clathrates. arXiv: 1901.04001v1 [cond-mat.supr.con], 1-5.

[35] Otto, H.H. (2008) Family Tree of Perovskite-Related Superconductors. arXiv: 0810.3501v1 [cond-mat.supr-con], 1-8. 


\section{Addendum}

1) It is believed that lanthanum (yttrium) strips off three electrons and can therefore be termed a cation regardless of whether the formed super-hydride is classified as metallic. From La-H distances of $\mathrm{LaH}_{10}$, being about $2.12 \AA$ respectively $2.21 \AA$, the radius of the highly coordinated lanthanum supports the picture of a "cation" with oxidation state $3+$ and ionic radius $r_{[32]}=d(\mathrm{La}-\mathrm{H})-$ $0.5 \cdot d(\mathrm{H}-\mathrm{H}) \approx 1.6 \AA$, while the covalent radius is already $2.07 \AA$.

2) There is a clear agreement as to denote zeolite cages or clathrate ones. Hydrogen clathrates of yttrium should serve as examples. The crystal structure of $\mathrm{YH}_{3}$ is formed by cages of 14 hydrogen atoms around $\mathrm{Y}$, showing a regular

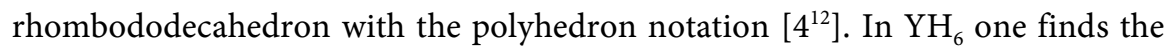
cube-truncated octahedron as cage, a combination of the octahedron with the cube, assembling 24 hydrogen atoms, polyhedron notation $\left[4^{6} 6^{8}\right]$. Only this cage is named after the mineral sodalite. Finally, in $\mathrm{YH}_{10}$ the hydrogen cage is a cube-truncated rhombododecahedron, a combination of the rhombic dodecahedron with the cube, polyhedron notation $\left[4^{6} 6^{12}\right]$. Therefore, you need not classify all cages as sodalite-like.

3) Supporting data for verifying Equation (7).

Table A. Information about conventional superconductors.

\begin{tabular}{|c|c|c|c|c|c|c|c|c|}
\hline \multirow{2}{*}{ Compound } & \multirow{2}{*}{$\begin{array}{l}\text { Space } \\
\text { Group }\end{array}$} & \multirow{2}{*}{$a(\AA)$} & \multirow{2}{*}{$c(\AA)$} & \multirow{2}{*}{$d(\AA)$} & \multirow{2}{*}{$\sigma$} & \multicolumn{2}{|c|}{$T_{\mathrm{c}}(\mathrm{K})$} & \multirow{2}{*}{ Ref. } \\
\hline & & & & & & $\exp$ & calc & \\
\hline $\mathrm{Rb}_{0.28} \mathrm{WO}_{3}$ & \multirow{2}{*}{ hexagonal } & 7.4 & 7.6 & 4.272 & 4.91 & 2.0 & 1.8 & [1] \\
\hline $\mathrm{K}_{0.3} \mathrm{ReO}_{3}$ & & 7.335 & 7.48 & 4.244 & 4.85 & 3.6 & 1.9 & {$[2]$} \\
\hline $\mathrm{K}_{0.5} \mathrm{MoO}_{3}$ & tetragonal & 12.36 & 3.86 & 3.86 & 4.33 & 4.2 & 3.5 & [2] \\
\hline $\mathrm{BaPb}_{0.75} \mathrm{Bi}_{0.25} \mathrm{O}_{3}$ & \multirow{2}{*}{$\operatorname{Pm} \overline{3} \mathrm{~m}$} & 4.303 & & 4.303 & 3.0 & 19 & 17 & [3] \\
\hline $\mathrm{Ba}_{0.6} \mathrm{~K}_{0.4} \mathrm{BiO}_{3-\delta}$ & & 4.293 & & 4.293 & 3.0 & 29 & 17 & {$[4]$} \\
\hline $\mathrm{Sr}_{0.86} \mathrm{Nd}_{0.14} \mathrm{CuO}_{2}$ & $\mathrm{P} 4 / \mathrm{mmm}$ & 3.942 & 3.38 & 3.38 & 2.0 & 43 & 41 & [5] \\
\hline $\mathrm{H}_{3} \mathrm{~S}$ & $\operatorname{Im} \overline{3} \mathrm{~m}$ & 2.984 & & 2.584 & 0.667 & 206 & 205 & [6] \\
\hline $\mathrm{YH}_{6} 150 \mathrm{GPa}$ & $\operatorname{Im} \overline{3} \mathrm{~m}$ & 3.605 & & 2.400 & 0.5 & 260 & 260 & [7] \\
\hline $\mathrm{LaH}_{10} 150 \mathrm{GPa}$ & \multirow{2}{*}{$\mathrm{Fm} \overline{3} \mathrm{~m}$} & 5.102 & & 2.551 & 0.3 & 286 & 299 & [8] \\
\hline $\mathrm{YH}_{10} 150 \mathrm{GPa}$ & & 4.800 & & 2.400 & 0.3 & 326 & 318 & [7] \\
\hline $\mathrm{LaH}_{12}$ & $\mathrm{Fd} \overline{3} \mathrm{~m}$ & 6.644 & & 2.35 & 0.25 & - & 341 & {$\left[{ }^{*}\right]$} \\
\hline
\end{tabular}

${ }^{\star}$ This work.

\section{References for Table A}

[1] Sweedler, A.R., Raub, Ch.J. and Matthias, B.T. (1965) Superconductivity of the Alkali Tungsten Bronzes. Physics Letters, 15, 108-109. https://doi.org/10.1016/0031-9163(65)91292-8

[2] Sleight, A.W., Bither, T.A. and Bierstedt, P.E. (1969) Superconducting Oxides of Rhenium and Molybdenum with Tungsten Bronze Type Structures. Solid State Communications, 7, 299-300. https://doi.org/10.1016/0038-1098(69)90404-9 
[3] Sleight, A.W., Gillson, J.L. and Bierstedt, P.E. (1975) High-Temperature Superconductivity in the $\mathrm{BaPb}_{1-\mathrm{x}} \mathrm{Bi}_{\mathrm{x}} \mathrm{O}_{3}$ Systems. Solid State Communications, 17, 27-28. https://doi.org/10.1016/0038-1098(75)90327-0

[4] Matheiss, L.F., Gyorgi, E.M. and Johnston Jr., D.W. (1988) Superconductivity above $20 \mathrm{~K}$ in the Ba-K-Bi-O) System. Physical Review B, 37, 3745-3746. https://doi.org/10.1103/PhysRevB.37.3745

[5] Smith, M.G., Manthiram, A., Zhou, J., Goodenough, J.B. and Markert, J.T. (1991) Electron-Doped Superconductivity at $40 \mathrm{~K}$ in the Infinite-Layer Compound $\mathrm{Sr}_{1-\mathrm{y}} \mathrm{Nd}_{\mathrm{y}} \mathrm{CuO}_{2}$. Nature, 351, 549-551. doi.org/10.1038/351549a0

[6] Duan, D.F., Liu, Y.X., Tian, F.B., Li, D., Huang, X.L., Zhao, Z.L., Yu, H.Y., Liu, B.B., Tian, W.J. and Cui, T. (2014) Pressure-Induced Metallization of Dense $\left(\mathrm{H}_{2} \mathrm{~S}\right)_{2} \mathrm{H}_{2}$ with High- $T_{c}$ Superconductivity. Scientific Reports, 4, 1-6.

https://doi.org/10.1038/srep06968

[7] Heil, C., di Cataldo, S., Bachelet, G.B. and Boeri, L. (2019) Superconductivity in Sodalite-Like Yttrium Hydride Clathrates. arXiv: 1901.04001v1 [cond-mat.supr.con], $1-5$.

[8] Semenok, D.V., Kruglov, I.A., Kvashnin, A.G. and Oganov, A.R. (2018) On Distribution of Superconductivity in Metal Hydrides. arXiv: 1806.00865 [cond-mat.supr.con], $1-19$, S1-S26. 